\title{
Abdomen agudo en el embarazo. Evaluación por resonancia magnética
}

\author{
Dres. Daniela Barahona $Z^{(1)}$, Giancarlo Schiappacasse $F^{(2)}$, Andrés Labra $W^{(2)}$, Pablo Soffia $\mathbf{S}^{(3)}$.
}

1. Radióloga. Facultad de Medicina Universidad del Desarrollo - Clínica Alemana de Santiago. Chile.

2. Profesor Adjunto de Radiología. Facultad de Medicina Universidad del Desarrollo - Clínica Alemana de Santiago. Chile.

3. Profesor de Radiología. Facultad de Medicina Universidad del Desarrollo - Clínica Alemana de Santiago. Chile.

\section{Acute abdomen in pregnancy. Magnetic Resonance evaluation}

\begin{abstract}
Acute abdominal pain in pregnant patients is a common reason for emergency consultation. Diagnosis is particularly difficult because of misleading factors related to normal pregnancy. Ultrasonography (US) in the evaluation of these patients is the first choice; the absence of radiation and side effects to the fetus allow its use without contraindications. However, the evaluation is limited by the normal anatomy alterations due to the displacement of the intra-abdominal structures, requiring greater skill of the operator and results are often uncertain. Given this scenario Magnetic Resonance Imaging (MRI) plays an increasingly important role, the absence of ionizing radiation and evidence of fetal harm, has allowed it to be incorporated as a tool when there is diagnostic uncertainly. This becomes especially important in suspected surgical pathology, acute appendicitis being one of the most common. MRI enables its identification as well as its differential diagnoses.

Keywords: Acute abdomen in pregnant patients, Magnetic resonance imaging in abdominal pain, MRI in pregnant patients.
\end{abstract}

Resumen: En las pacientes gestantes el dolor abdominal agudo es motivo común de consulta de urgencia. Su diagnóstico es particularmente difícil debido a factores confundentes relacionados al embarazo normal. La ultrasonografía (US) en la evaluación de estas pacientes es de primera línea; la ausencia de radiación y efectos secundarios en el feto permiten su uso sin contraindicaciones. Sin embargo, la evaluación se ve limitada por la alteración de la anatomía habitual debido al desplazamiento de las estructuras intra-abdominales, requiriendo mayor destreza del operador y resultados frecuentemente inciertos. Ante este escenario la resonancia magnética (RM) juega un rol de importancia creciente, la ausencia de radiación ionizante y de evidencias de daño fetal, han permitido incorporarla como herramienta ante dudas diagnósticas. Este hecho adquiere especial importancia en la sospecha de patología quirúrgica, siendo la apendicitis aguda una de las más frecuente. La RM permite reconocerla así como también a sus diagnósticos diferenciales.

Palabras clave: Abdomen agudo en embarazadas, Resonancia magnética en dolor abdominal, Resonancia magnética en embarazadas.

Barahona D. Abdomen agudo en el embarazo. Evaluación por resonancia magnética. Rev Chil Radiol 2015; 21(2): 70-75. Correspondencia:Dra. Daniela Barahona Z / danibarahona@gmail.com

Trabajo recibido el 27 de abril de 2015. Aceptado para publicación el 06 de julio de 2015.

\section{Introducción}

Frecuentemente las pacientes gestantes en el curso de su embarazo presentan dolor abdominal que requiere atención médica. Diferentes factores confundentes dificultan la evaluación clínica: leucocitosis inespecífica, desplazamiento de las estructuras intra-abdominales por el útero grávido, compresión ureteral por este último, náuseas y vómitos, dolor cólico por contracción uterina, etc. La patología que más frecuentemente requiere cirugía de urgencia es la apendicitis aguda, diagnóstico que se puede descartar mediante la utilización de RM. Ésta permite la evaluación adicional de numerosas estructuras abdominales sin utilización de radiación ionizante ni perjuicios demostrados tanto a la madre como al feto.

\section{RM en embarazo}

Bajo el conocimiento actual, no se han demostrado efectos deletéreos en el feto secundario al uso de la RM. El Colegio Americano de Radiología (ACR) aprueba el uso de imágenes de resonancia en pacientes embarazadas en cualquier trimestre de la gestación. Las ondas de radiofrecuencia no emiten radiación ionizante pero sí depositan su energía en forma de calor. Aunque la 
elevación de la temperatura fetal y del líquido amniótico no alcanzaría los niveles teratogénicos, el riesgo potencial debería ser considerado. Por estas razones siempre se requiere la evaluación de riesgos versus beneficios y en caso de no existir la necesidad de evaluación inmediata de la paciente, posponerla posterior al parto.

Los equipos de RM cuentan con mediciones de la energía depositada, pero utilizando estándares en base a pacientes no embarazadas. Estudios con modelos han demostrado que la mayor elevación de temperatura sería alcanzada en la madre y no en el feto(1).

La ACR no recomienda la utilización de medio de contraste (Gadolinio) durante la realización del estudio, ya que éste atraviesa la barrera placentaria ${ }^{(1)}$.

\section{Técnica de resonancia}

Se recomienda la realización del estudio en resonadores de 1,5 Tesla, ya que los de mayor campo magnético pueden generar mayores alzas en la temperatura. El estudio se realiza con la paciente en posición supina con una antena de superficie cubriendo el abdomen y la pelvis. El campo de estudio se debe extender desde el domo hepático hasta la sínfisis del pubis. El protocolo incluye adquisición de imágenes en los distintos planos (coronal, sagital y axial) en secuencias ponderadas en T2 Single-Shot Fast Spin-Echo, secuencias sensibles a líquido (T2 con saturación de la grasa) en corte axial, secuencias rápidas Estado Estacionario (steady-state) en corte axial, secuencias gradiente ponderadas en T1 dentro y fuera de fase en corte axial y secuencia difusión en adquisición axial(2) (Tabla I).

Las secuencias multiplanares permiten reconocer la localización del apéndice cecal. La secuencia sensible a líquido realza el edema peri-apendicular y en relación a cualquier estructura que presente cambios inflamatorios, además de evidenciar con facilidad el líquido libre. Las secuencias rápidas steady-state muestran las estructuras vasculares de alta señal pudiendo distinguirlas de estructuras tubulares como el apéndice cecal, además de evaluar su permeabilidad, a pesar de ser un estudio no contrastado. La secuencia ponderada en difusión muestra alta señal en las estructuras que presentan restricción a la difusión libre del agua como es el caso de los procesos inflamatorios y tumorales. Finalmente las secuencias gradiente ponderadas en T1 pueden confirmar la presencia de aire en el lumen apendicular, lo que disminuye la probabilidad de patología inflamatoria(3).

\section{Apendicitis aguda}

La evaluación de la apendicitis comienza con su localización. Debemos recordar que durante el segundo y tercer trimestre del embarazo el crecimiento uterino desplaza las estructuras pelvianas

\begin{tabular}{|c|c|c|c|c|c|c|c|}
\hline \multirow{2}{*}{$\begin{array}{l}\text { Parámetros } \\
\text { Tipo de secuencia }\end{array}$} & \multicolumn{3}{|c|}{ Single-Shot Fast SE T2 } & \multirow{3}{*}{$\begin{array}{l}\text { Single-Shot Fast } \\
\text { SE T2 con } \\
\text { saturación grasa } \\
\text { De disparo único } \\
\text { Axial }\end{array}$} & \multirow{3}{*}{$\begin{array}{l}\begin{array}{l}\text { Secuencia T1 } \\
\text { dentro y fuera } \\
\text { de fase }\end{array} \\
\text { Gradiente } \\
\text { Axial }\end{array}$} & \multirow{3}{*}{$\begin{array}{l}\text { Steady State } \\
\text { Free } \\
\text { Precession } \\
\text { Steady State } \\
\text { Axial }\end{array}$} & \multirow{3}{*}{$\begin{array}{l}\text { Difusión } \\
\text { Gradiente } \\
\text { Axial }\end{array}$} \\
\hline & De disp & aro único & & & & & \\
\hline Orientación & Axial & Coronal & Sagital & & & & \\
\hline \begin{tabular}{|l} 
Tiempo de \\
repetición (msec)
\end{tabular} & 717 & 833 & 833 & $800-1100$ & 175 & 3.7 & \\
\hline $\begin{array}{l}\text { Tiempo de eco } \\
\text { (msec) }\end{array}$ & 90 & 90 & 90 & 90 & $2,2-4,5$ & Mínimo & Mínimo \\
\hline $\begin{array}{l}\text { Grosor de corte } \\
(\mathrm{mm})\end{array}$ & 5 & 5 & 5 & 5 & 5 & 5 & 5 \\
\hline Gap (mm) & 0.5 & 1 & 1 & 0.5 & 0.5 & 0.5 & 0.5 \\
\hline $\begin{array}{l}\text { Campo de visión } \\
(\mathrm{mm})\end{array}$ & 310 & 420 & 420 & 310 & 310 & 310 & 310 \\
\hline $\begin{array}{l}\text { Número de } \\
\text { secciones }\end{array}$ & 66 & 34 & 27 & 66 & 66 & 36 & 66 \\
\hline Matriz & $288 \times 192$ & $384 \times 224$ & $384 \times 224$ & $320 \times 224$ & $288 \times 224$ & $224 \times 288$ & $100 \times 128$ \\
\hline
\end{tabular}


y el apéndice frecuentemente es desplazado a una posición superior a la habitual, pudiendo localizarse en el flanco o hipocondrio derechos, en posición subhepática. La localización del apéndice cecal en RM es de mayor dificultad que en tomografía computada, esto es aún mayor en el caso de evaluadores poco experimentados en la técnica. Se debe identificar el polo cecal y luego la estructura tubular que se origina de él y que termina en fondo ciego, al igual que en tomografía computada (TC). De especial utilidad es la secuencia sagital, que permite evaluar el ascenso del polo cecal y posterior identificación del apéndice vermicular.

Una vez localizado el apéndice se deben evaluar sus características: diámetro, grosor parietal, contenido y tejido adiposo peri-apendicular. El apéndice normal tiene un diámetro de hasta $6 \mathrm{~mm}$, grosor parietal de hasta $2 \mathrm{~mm}$, baja señal de su contenido en las secuencias T1 y T2, no deben existir alteraciones en la señal de la grasa peri-apendicular así como tampoco líquido libre y no debe presentar restricción a la difusión del líquido ${ }^{(3)}$ (Figura 1a,b).

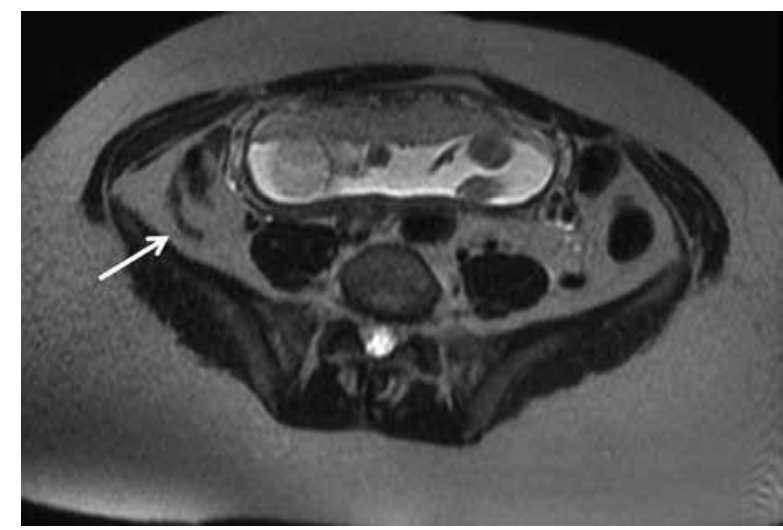

Figura 1a. Paciente de 39 años con embarazo de 29 semanas. Secuencia T2 axial donde se observa el apéndice cecal de calibre normal, con la señal habitual de la grasa periapendicular.

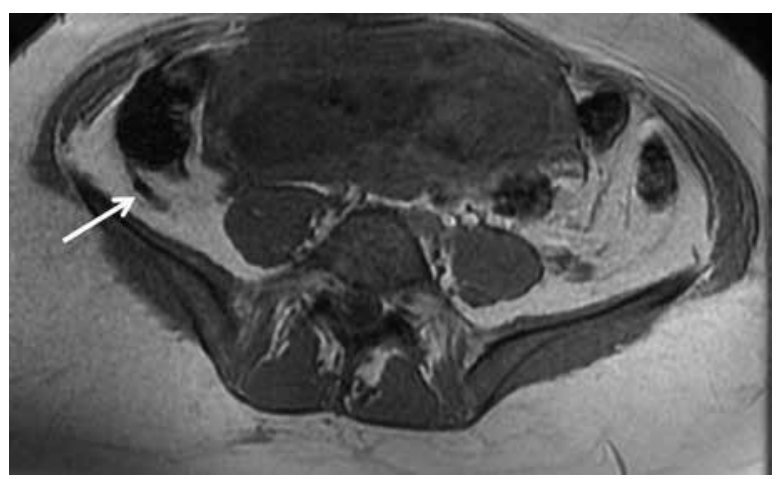

Figura 1b. Secuencia gradiente ponderada en T1 en fase que demuestra artefacto de "blooming" en el lumen apendicular, secundario a contenido aéreo intraluminal. Este hallazgo junto al calibre apendicular normal y la ausencia de cambios inflamatorios periapendiculares descartan la sospecha de apendicitis.
Las características del apéndice alterado son: diámetro mayor a $7 \mathrm{~mm}$, grosor parietal mayor a 2 $\mathrm{mm}$, contenido luminal de alta señal T2, hiperintensidad T2 de la señal de la grasa peri-apendicular asociado a la presencia de líquido y restricción a la difusión. Si el apéndice mide entre 6 y $7 \mathrm{~mm}$ de diámetro, presenta contenido de alta señal T2 pero no se identifican cambios inflamatorios en el tejido adiposo o engrosamiento de sus paredes, se debe realizar seguimiento de la paciente, al ser los hallazgos no concluyentes (Figura 2a,b).

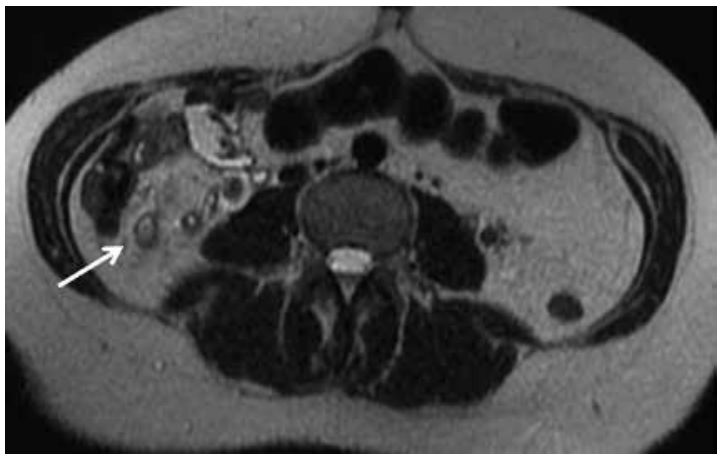

Figura 2a. Paciente de 35 años con embarazo de 14 semanas. Secuencia T2 axial donde se observa el apéndice cecal dilatado, con leve engrosamiento de su pared y tenue edema de la grasa periapendicular compatible con appendicitis aguda.

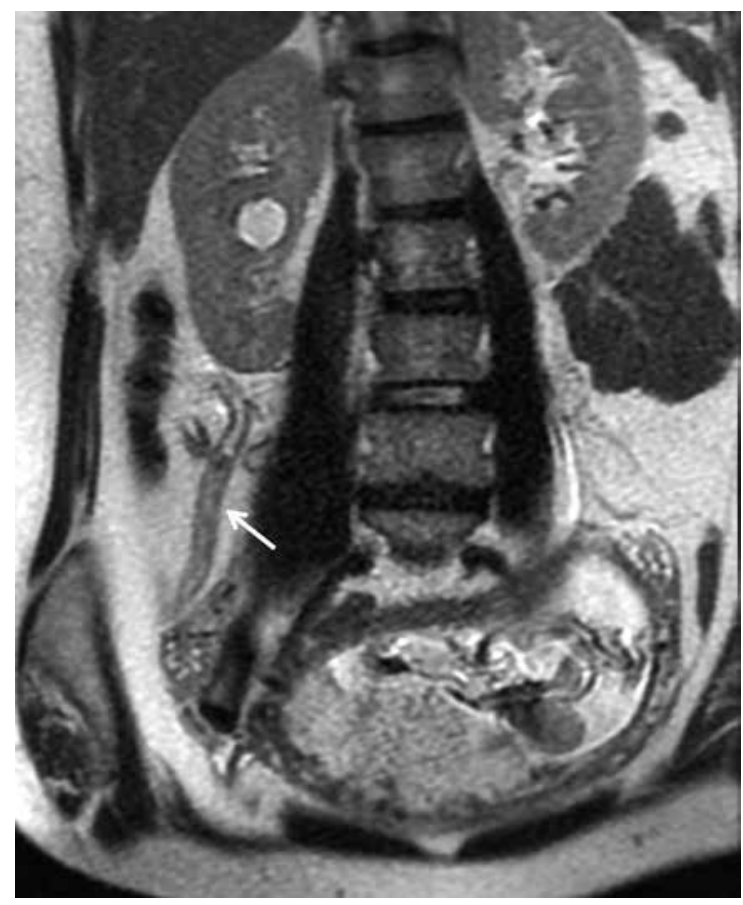

Figura 2b. Secuencia T2 coronal donde se observa el apéndice cecal dilatado en todo su trayecto, con engrosamiento de su pared y leve edema periapendicular en su tercio craneal.

Se ha descrito una sensibilidad de $100 \%$ y especificidad de $94 \%$ en el diagnóstico de apendicitis durante el embarazo mediante $\mathrm{RM}^{(4)}$. 


\section{Otros desórdenes gastro-intestinales}

En el estudio de la paciente embarazada con dolor abdominal podemos encontrar otras patologías inflamatorias gastro-intestinales, además de la apendicitis aguda, algunas de ellas también localizadas en la fosa ilíaca derecha, como la ileítis distal, diverticulitis de colon derecho, colitis derecha y el infarto omental.

La RM permite evaluar con muy buena precisión la pared del colon e intestino delgado. El edema parietal se reconoce como engrosamiento y aumento de la señal T2 de la pared, así como también los cambios inflamatorios en el tejido adiposo adyacente se manifiestan como aumento de la señal T2. La RM presenta una sensibilidad superior a la TC en la detección del edema parietal (Figura 3a,b).

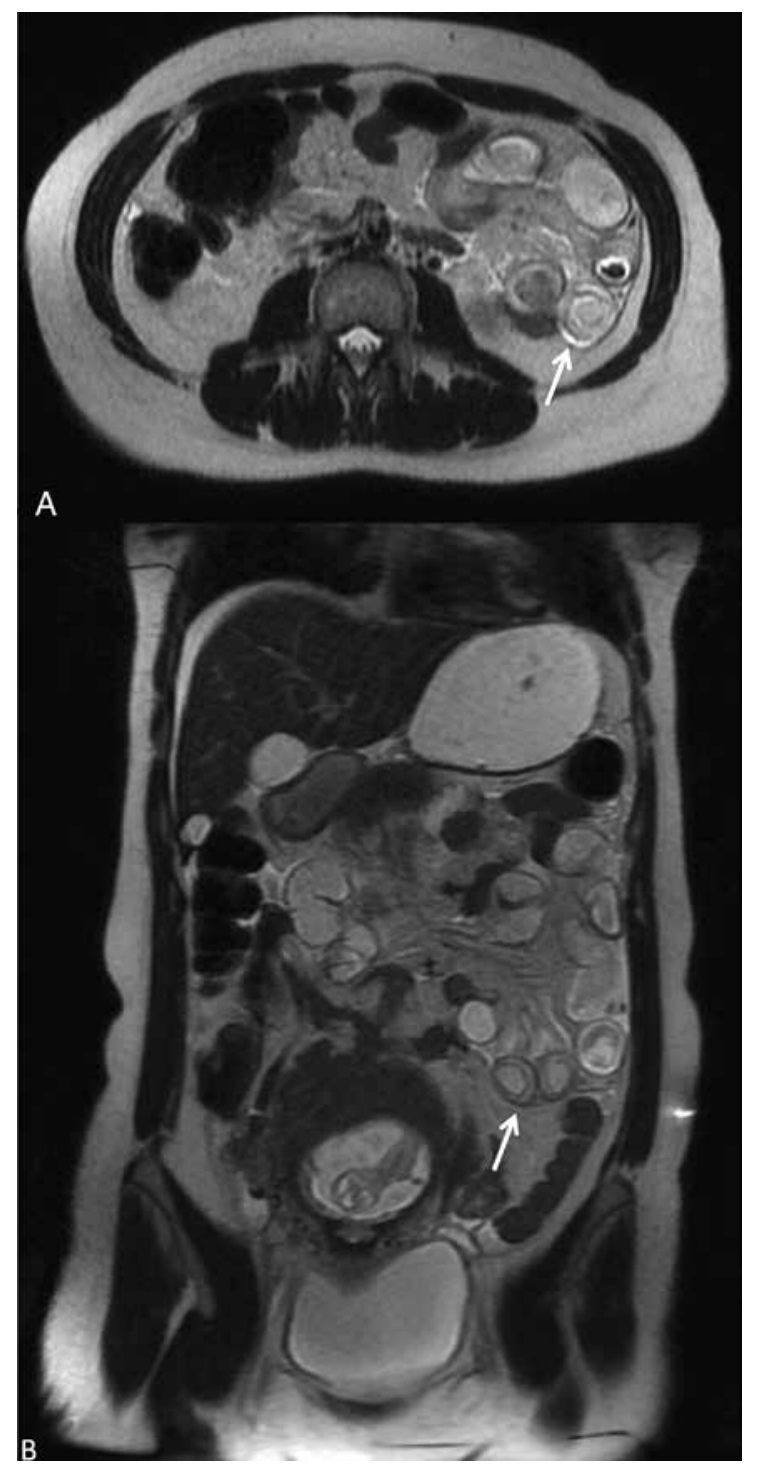

Figura 3a, b. Paciente de 42 años con embarazo de 12 semanas. Secuencia T2 axial y coronal que demuestra dilatación de asas de intestino delgado en el flanco izquierdo, engrosamiento parietal con morfología en "diana" e hiperseñal T2 de la grasa adyacente secundario a la presencia de líquido, compatible con enteritis.
Las complicaciones de una enfermedad de Crohn tales como los trayectos fistulosos o formación de abscesos también son bien evaluados con la RM.

En la población general la RM tiene una sensibilidad de $91 \%$ y especificidad de $71 \%$ en la detección de enfermedad inflamatoria intestinal activa ${ }^{(5)}$.

Los procesos inflamatorios flegmonosos se visualizan como tejido de partes blandas de señal intermedia en T1 y alta señal T2 y cuándo éste forma parte de un absceso presenta características de señal similares al líquido.

La capacidad multiplanar del estudio de RM permite evaluar el punto de cambio de calibre en el caso de una obstrucción intestinal y eventualmente determinar su causa, al igual que la TC, sin la necesidad del uso de medio de contraste intravenoso (Figura 4a,b).

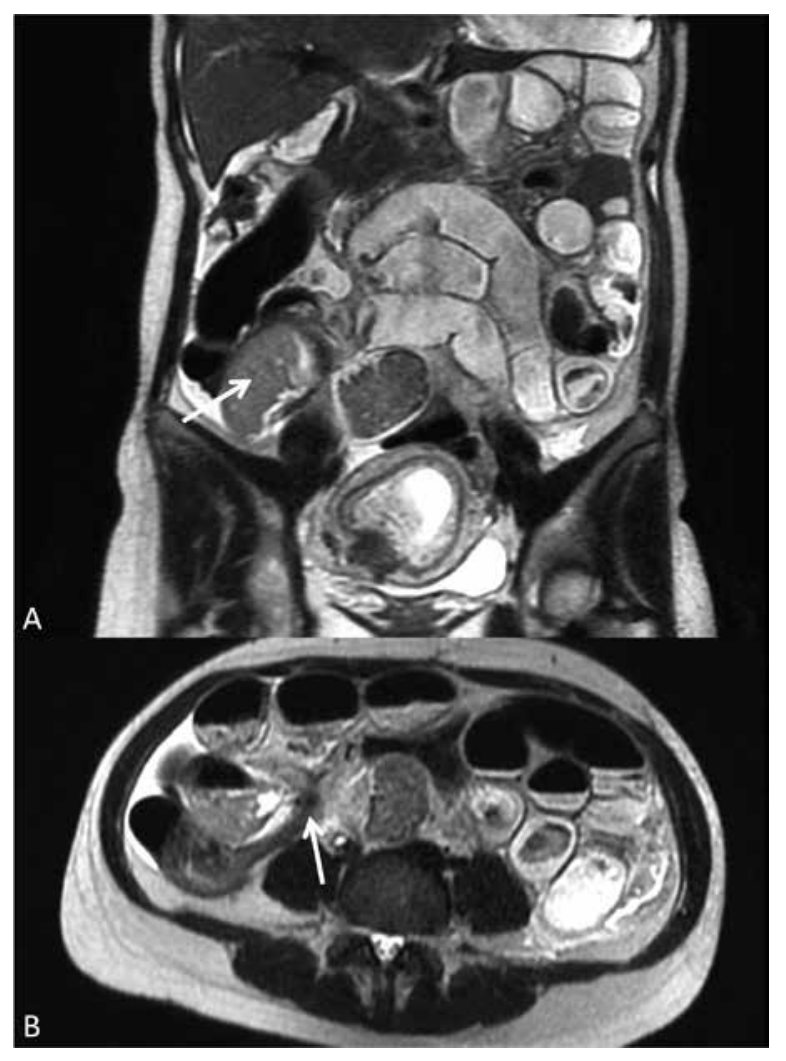

Figura 4. Paciente de 33 años con embarazo de 9 semanas que consulta por dolor abdominal. US previo demuestra dilatación de asas intestinales. Corte coronal de secuencia T2 que confirma la dilatación difusa de las asas de intestino delgado, con formación de pseudoheces (flecha) próximo al sitio de obstrucción. Corte axial de secuencia T2 que muestra asas intestinales traccionadas (flecha), incluyendo el apéndice cecal, en la fosa ilíaca derecha, compatible con obstrucción intestinal mecánica secundario a bridas.

En el caso de hernias de la pared abdominal, la excelente resolución por contraste de la RM, permite la evaluación del defecto herniario y de los posibles signos de complicación. 
En la diverticulitis aguda la RM puede identificar el divertículo involucrado que se asocia a engrosamiento mural de la pared colónica comprometida, hiperseñal T2 del tejido adiposo adyacente y líquido libre. También se pueden detectar las complicaciones como la formación de colecciones, fístulas y perforación. En la población general la detección de la diverticulitis por RM se ha reportado una sensibilidad de $86-94 \%$ y especificidad de $88-92 \%{ }^{(6)}$.

\section{Causas hépato-biliares}

Las causas hepatobiliares de dolor abdominal incluyen colelitiasis, coledocolitiasis, colecistitis aguda, pancreatitis y hepatitis. Estas patologías son frecuentes en mujeres en edad reproductiva.

La colelitiasis es fácilmente evaluada mediante $\mathrm{RM}$, donde se reconocen defectos de baja señal T2 en el lumen vesicular. Si éste se asocia a engrosamiento de la pared vesicular, dilatación del lumen y edema del tejido adiposo debemos plantear una colecistitis aguda ${ }^{(3)}$.

La coledocolitiasis igualmente se detecta como un defecto de baja señal T2 en el lumen de la vía

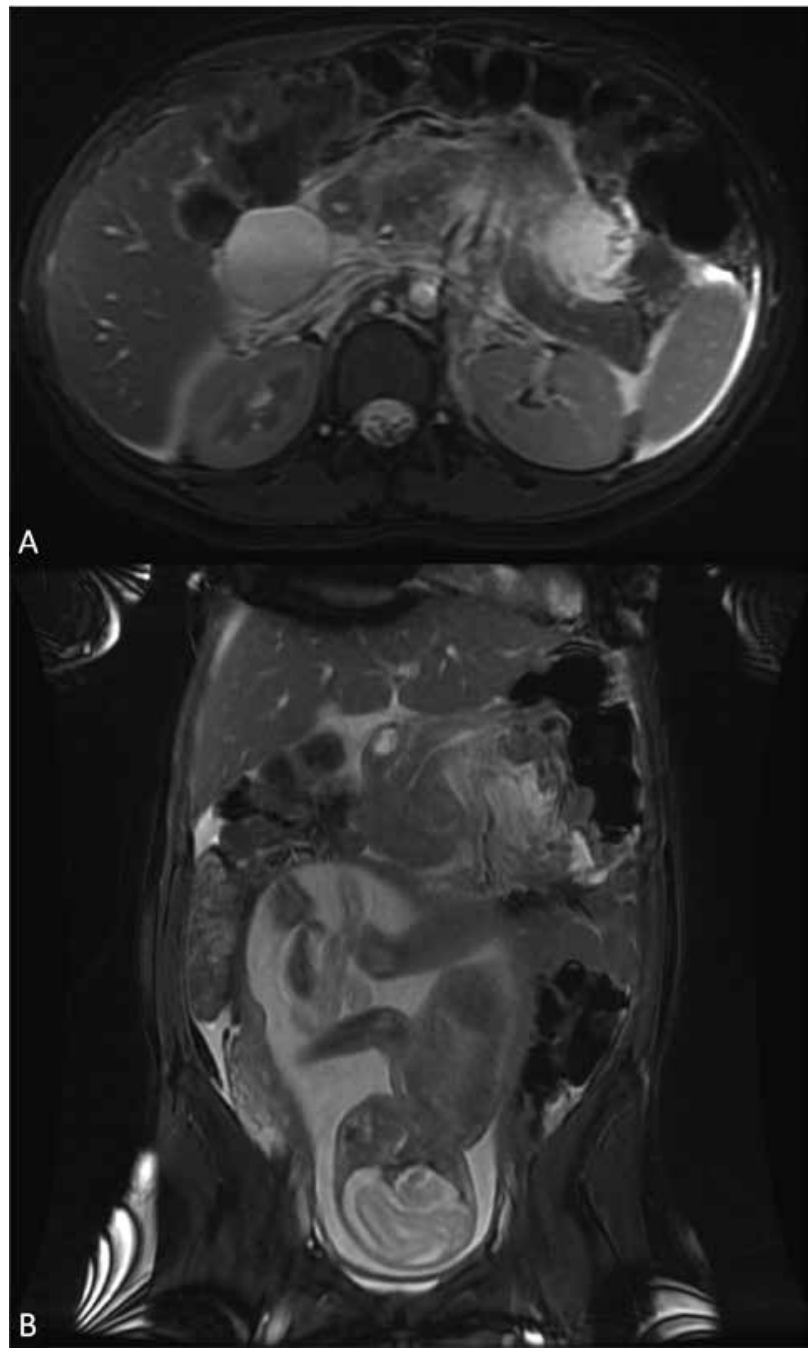

biliar, frecuentemente asociado a dilatación de la vía biliar intra y extrahepática.

La pancreatitis aguda se manifiesta como edema del páncreas caracterizado por aumento de su tamaño y de la señal en T2, además de presencia de señal de líquido en el espacio peri-pancreático. Podremos a su vez detectar una posible causa biliar (Figura 5a,b).

El síndrome de HELLP en el contexto de paciente con preeclampsia o eclampsia severa se puede presentar con hepatomegalia, edema hepático caracterizado por áreas de aumento de la señal en T2 del parénquima, ascitis y derrame pleural. También podemos evaluar sus complicaciones como los hematomas hepáticos y peri-hepáticos ${ }^{(3,7)}$.

El hígado graso agudo usualmente aparece durante el tercer trimestre del embarazo y en RM se puede presentar con hepatomegalia y esteatosis difusa, detectada por la caída de señal del parénquima hepático en la secuencia ecogradiente T1 fuera de fase ${ }^{(3,7)}$.

\section{Causas genito-urinarias}

La obstrucción del tracto urinario es una causa frecuente de dolor abdominal durante el embarazo. Un factor confundente es la dilatación fisiológica del sistema excretor renal, frecuentemente del lado derecho, debido a factores hormonales y a la compresión uterina extrínseca y de los vasos gonadales (prominentes durante la gestación) contra el músculo psoas. La dilatación fisiológica ocasionalmente puede producir dolor y es importante diferenciarla de una obstrucción por cálculo(3,7).

En RM la obstrucción se manifiesta por aumento del tamaño renal, líquido perinéfrico y cambio de calibre abrupto del lumen ureteral. Cuando es visible el cálculo se observa como un defecto de baja señal en el lumen ureteral. Esta modalidad no es de primera línea en la evaluación de la uréterolitiasis dado su limitada evaluación de cálculos pequeños.

La pielonefritis aguda se puede manifestar como aumento del tamaño renal, áreas de edema y restricción a la difusión en el parénquima y aumento de la señal en T2 del espacio perirrenal. Además se puede evaluar la presencia de colecciones de señal líquida intra o perirrenales (Figura 6a,b).

Figura 5. Paciente de 31 años cursando embarazo de 26 semanas con abdomen agudo. Corte axial de secuencia FISP con saturación grasa que muestra aumento de la intensidad de señal del cuerpo del pánceas, abundante líquido libre libre intraabdominal compatible con pancreatitis aguda. Corte coronal de secuencia FISP con saturación grasa que confirma los hallazgos descritos y muestra el útero grávido. 


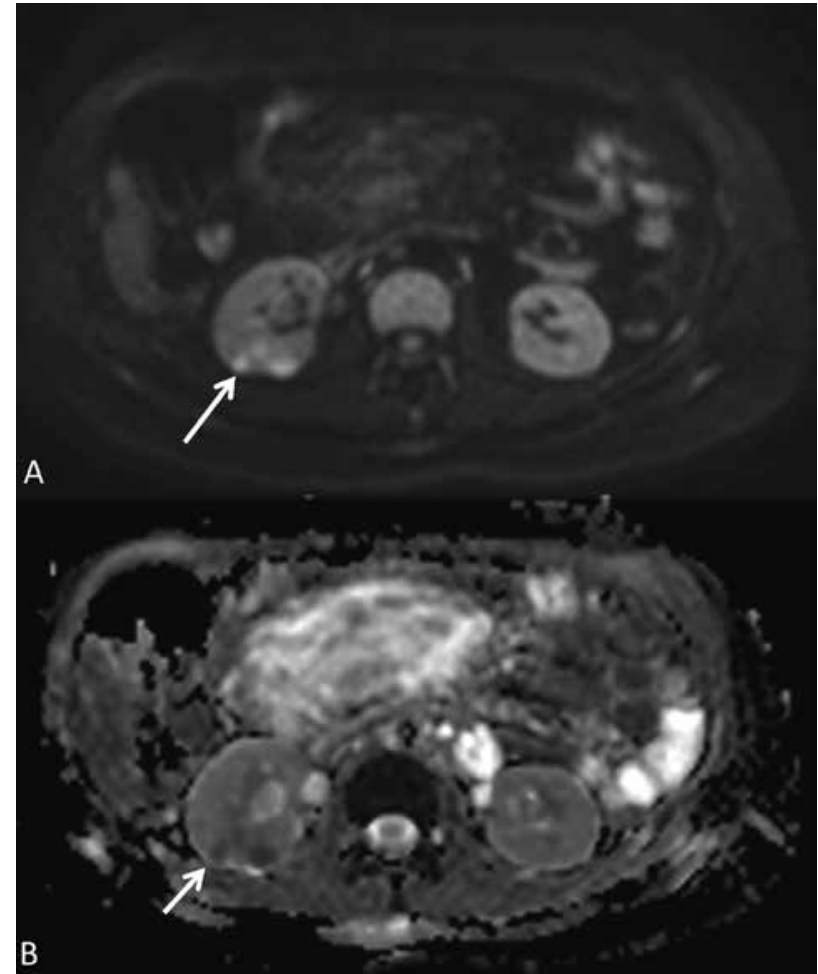

Figura 6. Paciente de 21 años con embarazo de segundo trimestre que consulta por dolor abdominal. Secuencia difusión que demuestra áreas de restricción en el borde posterior del parénquima renal derecho compatible con focos de nefritis. Secuencia ADC que confirma el hallazgo de nefritis derecha, no visible en las restantes secuencias.

\section{Causas ginecológicas}

Dentro de las causas ginecológicas se encuentran los leiomiomas complicados, las torsiones de lesiones anexiales o de los mismos anexos y la enfermedad inflamatoria pelviana.

Los leiomiomas habitualmente muestran señal en T1 heterogénea pero cuando se complican pueden presentar focos de hemorragia o áreas de señal líquida debido a necrosis, pudiendo provocar dolor, alteraciones que pueden ser visibles en RM.

La torsión ovárica ocurre con mayor frecuencia en el primer trimestre del embarazo y tiene una prevalencia similar a la apendicitis aguda en el embarazo (1/1800). Las características de la torsión incluyen el aumento de volumen ovárico, localización inhabitual y edema del tejido adiposo que lo rodea. Puede reconocerse en ciertas ocasiones la torsión del pedículo vascular(2,7).

El embarazo disminuye el riesgo de un proceso inflamatorio pelviano, en el caso de sospecha se puede reconocer como dilatación con líquido de las trompas uterinas, colecciones líquidas anexiales secundario a la presencia de abscesos y aumento de la señal T2 difusa del tejido adiposo pelviano, muchas veces asociado a engrosamiento del peritoneo pelviano.

\section{Conclusión}

El dolor abdominal agudo en la paciente gestante continúa siendo un desafío debido a los múltiples factores confundentes que se asocian al embarazo. La RM es una buena alternativa que se está utilizando como nueva herramienta en la evaluación de la apendicitis aguda con US negativa. Su buena sensibilidad y evaluación de numerosos otros órganos aportan a la confirmación diagnóstica y diagnóstico diferencial de innumerables patologías.

\section{Bibliografía}

1. Tremblay E, Thérasse E, Thomassin-Naggara I, Trop I. Guidelines for Use of Medical Imaging during Pregnancy and Lactation. Radiographics 2012; 32: 897-911.

2. Pedrosa I, Zeikus EA, Levine D, Rofsky NM. MR Imaging of Acute Right Lower Quadrant Pain in Pregnant and Nonpregnant Patients. Radiographics 2007; 27: 721-753.

3. Spalluto L, Woodfield C, DeBenedectis C, Lazarus E. MR Imaging Evaluation of Abdominal Pain during Pregnancy: Appendicitis and Other Nonobstetric Causes. Radiographics 2012; 32: 317-334.

4. Pedrosa I, Levine D, Eyvazzadeh AD, Siewert B, Ngo L, Rofsky NM. MR Imaging Evaluation of Acute Appendicitis in Pregnancy. Radiology 2006; 238: 89-899.

5. Koh DM, Miao Y, Chinn RJ, Amin Z, Zeegen R, Westaby D, et al. MR Imaging Evaluation of the Activity of Crohn's disease. AJR. Am J Roentgenol 2001; 177(6): 1325-1332.

6. DeStigter KK, Keating DP. Imaging Update: Acute Colonic Diverticulitis. Clin Colon Rectal Surg 2009; 22(3): 147-155.

7. Woodfield C, Lazarus E, Chen K, Mayo-Smith W. Abdominal Pain in Pregnancy: Diagnoses and Imaging Unique to Pregnancy-Review. AJR 2010 194: WS14WS30. 